\title{
Concurrent validity of the Wii Stillness Test as a measure of balance performance in older adults
}

\author{
Anton J. Simms BSª, Luis R. Hernandez MSEd ${ }^{a}$, Emerson Sebastião PhD ${ }^{\mathrm{a}, *}$ \\ ${ }^{a}$ Health and Exercise Research Group, Department of Kinesiology and Physical Education, \\ Northern Illinois University, DeKalb, IL, USA; *Corresponding author: esebastiao@niu.edu
}

\begin{abstract}
Background: Research grade laboratory force plates are considered the gold standard method of assessing balance performance. However, these can cost upwards of thousands of dollars. Discovering a more cost-effective way of assessing balance performance could prove to be beneficial to the older adult community. The Wii Stillness Test, a preprogrammed balance assessment test as part of Nintendo's Wii Fit exergame, may bring a solution in the form of providing an easily acquisitioned measure of balance performance. Objective: This study investigated the concurrent validity of the Wii Stillness Test (WST) as a measure of static balance performance in older adults by comparing WST scores with balance metrics collected via force platform.

Methods: Older adults without cognitive or lower body impairment were recruited. Participants completed 1 session of 630 -second dual-stance balance assessments: 3 on the Wii Balance Board using Wii Fit software and WST, 3 on the force platform. WST calculates a score (ST) while 95\% sway area (SA) and average sway velocity (SV) were calculated from the center of pressure data. All scores were averaged.

Results: A total of 26 older adults ( 16 females and 10 males), mean age $81.31 \pm 7.84$ years, and body mass index of $27.72 \pm 4.92 \mathrm{~kg} / \mathrm{m} 2$ were assessed. Spearman's rho correlations coefficients revealed a significant association between ST scores and 95\% SA ( $\rho=-.61 ; p=$ $001)$ and a marginal association between ST scores and SV ( $\rho=-.33 ; p=.096)$.

Conclusion: Our findings suggest the WST is a valid method of assessing static balance performance in older adults.
\end{abstract}

Keywords: Exergame, balance, older adults, validation

\section{INTRODUCTION}

Balance is an important clinical measure in a diseased and older adult population. This is based on evidence that poor balance skills are associated with increased fall risk (Al-Aama, 2011). Falls are a public health concern due to their consequences for the individual and related costs. Evidence suggests that 1 in 5 falls causes a serious injury such as broken bones or head injury (Alexander, Rivara, \& Wolf, 1992; Sterling, O'Connor, \& Bonadies. 2001). This is concerning as it poses a threat to the health of older adults and reduces their ability to remain independent, negatively affecting the quality of life (Trombetti et al., 2016). Further, data from the Centers for Disease Control and Prevention demonstrated that fall death rates in the U.S increased around $30 \%$ from 2007 to 2016 for older adults.

The ability to accurately assess balance is important as it can provide paramount information for clinicians and practitioners. Field-tests such as the Berg Balance Scale, although widely accepted, may lack precision as suggested by previous work conducted in the diseased population (La Porta et al, 2012; Blum \& Korner-Bitensky, 2008). Laboratory-based tests, for instance,the use of force plat- form to assess posturography are considered the gold standard technique for balance evaluation. This approach allows researchers to calculate center of pressure and its derived metrics such as sway area and sway velocity; metrics that are used to express balance performance. Although it is considered the gold standard technique, the use of such an instrument is expensive and requires high-specialized personnel to run and analyze the data. To this end, examining new tests that can provide accurate and valid balance information while being practical and accessible, not requiring expensive equipment, could be of great value in both clinical and community settings.

A more cost-effective way to assess the balance performance of older adults could be to utilize a Nintendo Wii video game console (Nintendo, Minami-ku Kyoto, Japan), the exercise-based video game (exergame) Wii Fit software, and Wii Balance Board (WBB) peripheral (a small rectangular board that acts similar to a force plate). The Wii Fit software comes with the Wii Stillness Test (WST). This test has the participant stand on the WBB as still as possible for 30 -seconds and gives a percentage score $(0-100 \%)$ with lower scores corresponding to worse balance and vice versa. 
The WBB itself as a tool has shown ability to be used to collect center of pressure (COP) data, providing comparable data to a force platform when analyzing path lengths during balance trials (Clark et al., 2010; Park \& Lee, 2014). However, collecting COP data from the WBB requires specialized software and personnel, limiting the utility of the WBB as a more practical alternative to force platforms. The use of the WST is relatively simple and requires no software beyond the Wii Fit software itself. Previous research has shown that the WST could differentiate between those who are at risk of falling from those who were not when accounting for age and gender (Jorgensen, Hansen, Perez, \& Spaich, 2014). It has been further observed that the WST has a high degree of reproducibility in older adults, as well as moderate to excellent concurrent validity (Jorgensen, Laessoe, Hendriksen, Nielsen, \& Aagaard, 2014; Negus et al., 2019). Despite providing valuable preliminary information regarding the validity of the WST, to the best of our knowledge, very few studies have been conducted with such a purpose. Due to the limited studies of this nature, it is important to investigate whether the WST and its corresponding score can be used to accurately assess static balance performance in different populations of older adults. This would help establish the WST as a valid measure of static balance among older adults. This would provide clinicians and community based-facilities a small, low budget, and simple tool to measure an important fitness component in this population. Therefore, this study examined the concurrent validity of the WST as a measure of static balance performance in older adults by comparing scores of this test with data collected through a force platform.

\section{MAterials AND METHODS Study design}

This cross-sectional study was approved by the Institutional Review Board of Northern Illinois University and informed consent forms were signed by all participants prior to testing.

\section{Participants}

Participants were recruited from a senior housing facility and the community in northern Illinois. Inclusion criteria included: (a) being at least 60-years-old; (b) reporting no lower body musculoskeletal injuries within the past 6 months; (c) able to stand without an assistive device; and (d) cognitive abilities to understand the proposed tasks. 27 older adults were recruited and enrolled in the study $(n=25$ from the senior housing facility and $n=2$ from the community). 1 participant was excluded due to hip osteoporosis and inability to stand for the test trials. The final analytical sample was 26 older adults.

\section{Measures}

Balance

Posturography was the criterion measure used to objectively assess balance using a force platform (Accusway, AMTI, Watertown, MA) and based on assessments of COP. Briefly, participants were asked to stand on the force platform for 30 seconds. Feet were angled out approximately 30 degrees with the heels separated $11 \mathrm{~cm}$ apart. A visual mark was placed at eye level on a wall 1.5 $\mathrm{m}$ in front of the participant. Participants completed 3 trials with eyes open and were given at least 30 seconds of seated rest between trials. The COP data was then exported to AMTI's Bioanalysis software (BioAnalysis, AMTI, Watertown, MA) for the calculation of $95 \%$ sway area (SA; $\mathrm{cm} 2$ ) and average sway velocity (SV; $\mathrm{cm} / \mathrm{sec}$ ).

\section{Wii Stillness Test (WST)}

Balance performance was also assessed using the WST. Participants were asked to stand on the WBB for 30 seconds. Feet were placed in the grooved area of the WBB with feet pointed straight forward. Normally the user looks at the screen as visual stimulus during the WST, but for this study, a visual mark was placed at eye level on a wall $1.5 \mathrm{~m}$ in front of the participant with the WST screen out of view. Participants were given at least 30 seconds of seated rest between trials. Concluding each trial, a stillness test score (ST) was given by the WST to indicate balance performance. Scores were given as a percentage from $0-100 \%$. Scores closer to 0 indicating poorer balance performance and scores closer to $100 \%$ better balance performance. After the 3 assessments, average ST was calculated.

\section{Other measures}

Demographic, health, and health behavior information was gathered for sample characterization purposes. Demographic data included age and sex. Health and health behavior data included height and weight (later used for body mass index calculation), reported number of medical conditions (later categorized into $0-1 ; 2-3 ;>3$ ), and minutes per week spent in physical activity or exercise. The last was measured using a self-report question that asks participants to describe type, frequency, duration, and intensity of regular physical activity or exercise they partake. Frequency and duration were later multiplied in order to calculate average minutes per week spent in physical activity or exercise.

\section{Procedure}

Assessments were conducted in the main office of the fitness center located in the senior housing facility and the biomechanics laboratory in the Department of Kinesiology and Physical Education at Northern Illinois University. All assessments were completed in a single session, 
Table 1. General demographic, health and health behavior characteristics of the sample.

$\mathbf{n}=\mathbf{2 6}$

Age (year)

$81.31 \pm 7.84$

Sex, \%

Female

$16(61 \%)$

Male

$10(39 \%)$

Height, $\mathrm{cm}$

$168.05 \pm 12.14$

Weight, Kg

$77.04 \pm 17.35$

$\mathrm{BMI}, \mathrm{kg} \cdot \mathrm{m}^{-2}$

$27.72 \pm 4.92$

Physical activity, minutes/week

$158 \pm 83$

Reported medical conditions, \%

$14(54 \%)$

$8(31 \%)$

$>3$

$4(15 \%)$

Note: values are mean and standard deviation unless otherwise noted.

lasting approximately 45 minutes. Participants read and signed the informed consent form then completed a demographic and health history questionnaire. Participant's body composition was then measured using bioelectrical impedance analysis (InBody 520; GE Healthcare Lunar, Madison, WI, USA). After body composition assessments, participants completed 630 -second dual-stance balance assessments: 3 on the Wii Balance Board (WBB) and Wii Fit software, and 3 on the force platform. The order of trials was randomized using a random number generator.

\section{Statistical analysis}

Statistical analyses were performed using SPSS version 24 (IBM Corporation, Armory, N.Y) and significance was set at $\mathrm{P}<.05$. Descriptive statistics were used to characterize the sample. The normality of the data was examined by the Shapiro-Wilk test. The test indicated significant violations in normality for SA $(\mathrm{W}(26)=.885, \mathrm{p}$ $=.007)$ and SV $(\mathrm{W}(26)=.880, \mathrm{p}=.006)$. We thus used Spearman's rho correlation analysis to assess the concurrent validity of the WST scores against balance metrics collected using the force platform. Interpretation was based off Cohens (1988) guidelines with values of $0.1,0.3$, and 0.5 represent small, moderate, and large correlations, respectively. 95\% confidence intervals were included for all correlations.

Table 2. Spearman rank correlations between WST scores and measures of balance collected through a force platform.

\begin{tabular}{lccc}
\hline Variable & WST Scores & $\mathbf{9 5 \%} \mathbf{C l}$ & $\boldsymbol{P}$-value \\
\hline Sway area $\left(\mathrm{cm}^{2}\right)$ & -.61 & $-.78 ;-.35$ & .001 \\
Sway velocity $(\mathrm{cm} / \mathrm{s})$ & -.33 & $-.64 ; .06$ & .096 \\
\hline
\end{tabular}

\section{Results}

Demographic and health information of the sample is provided in Table 1. Briefly, the average age of the participants was 81 years old with the majority being women. Participants were on average classified as overweight and reported an average of 158 minutes of physical activity or exercise per week, which mostly included walking or light intensity exercise focusing on improvements in activities of daily living.

\section{Correlations}

Spearman's rho correlations were used to test the association between WST scores and 95\% SA and SV. A significant $(\mathrm{P}<.05)$ strong inverse association was observed between WST scores and SA values in our sample (Table 2 and Figure 1); but a moderate non-significant inverse association was observed between WST scores and SV.

\section{Discussion}

This study investigated the concurrent validity of the Wii Stillness Test as a measure of static balance performance in older adults. WST scores were strongly and inversely associated with $95 \%$ SA. Although the WST scores were moderately and inversely associated with SV, such association was not found to be statistically significant (i.e. marginal). These findings partially corroborate our hypothesis that the WST scores would be moderate-to-strongly associated with established objective linear measures of balance.

In a study conducted by Jorgensen et al. (2014), the authors examined the preliminary reproducibility and validity of the WST scores as a measure of balance in 30 older adults (22 women, 8 men) with an average age of 72 years old. For the validation portion, the authors collected information on both the WST and different force platform variables including SA and SV. Four trials per test (i.e., WST and force platform) were performed for each participant. The findings reported in the study partially corroborate the results observed in the present study (Table 2 and Figure 1). They found a strong significant inverse association between WST scores and both SA and SV, whereas the present study found a strong significant inverse association only between WST and SA, and a moderate marginal significant (Table 2) between WST scores and SA. A partial explanation for the different results observed between the present study and the study conducted by Jorgensen et al. (2014) may be related to the low variance in the characteristic of our sample compared to the Jorgensen and colleagues. The participants in the previous were randomly recruited from senior citizens' clubs and society organizations in Aalborg, Denmark. In the present study, participants were recruited by convenience from one senior housing facility 

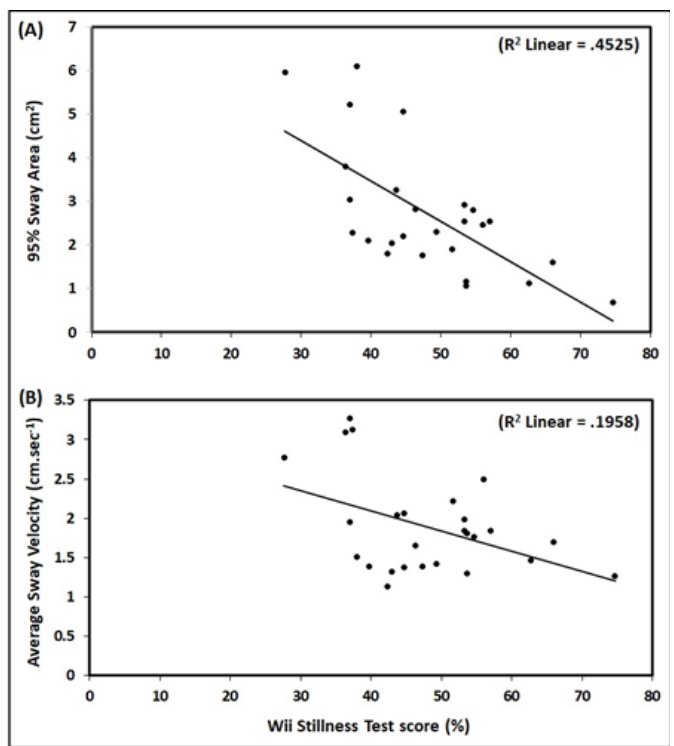

Figure 1. Scatterplot of the association between the Wii Stillness Test Scores and 95\% Sway Area (A); and Wii Stillness Test Scores and Average Sway Velocity (B).

in the Midwest area of United States (the majority of participants) and a local community. In addition, it is possible that the lower number of trials allowed in this study compared to Jorgensen and colleagues may be accounted for the difference in the results comparing WST scores and SA and SV. In the present study, participants performed three successful trials for each test (i.e., WST and force platform) compared to four trials performed by Jorgensen and colleagues. Nevertheless, both studies demonstrated that the WST scores is a valid measure of static balance within the older adult population.

The validity of the WST score has implications for research, clinical, and professional practice. Currently, the force platform derived information is considered the gold standard for measures of balance. Several field tests have been widely used in clinical settings; however, the use of force platforms and some clinical tests present several challenges. For example, a force platform is costly and calculating balance performance (SA \& SV) from COP data collected requires specialized knowledge, expensive software and is time-consuming. Further, as previously mentioned, some clinical tests such as the Berg scale may not be precise (La Porta et al, 2012; Blum \& Korner-Bitensky, 2008). To this end, the WST would be a feasible tool/test that could be used to complement well-established field tests widely adopted in clinical practice to provide more accurate information, for example, about balance in the older adult population.

This study comes with limitations. One potential limitation could be related to the method in which balance trials were conducted. During trials, subjects performed 6 separate standing balance assessments (i.e., 3 using the WST and 3 using the force platform). Other studies have opted to use a set up where the WBB is stacked on top of the force platform, and both devices were used simultaneously to assess balance in a single trial (Leach, Mancini, Peterka, Hayes, \& Horak, 2014; Negus et al., 2018). This approach could have eliminated between trial performance differences or reduce the amount of fatigue accumulated by the older adult. However, it is important to note that enough resting period between trials was provided to all participants, which we believed to be appropriate to mitigate fatigue. Other limitations lie in the small sample size. A strength of this study was the selection of the force platform derived balance metrics as the criterion measure. This approach is considered the gold standard method for balance assessment. Despite the limitation, we were able to engender additional evidence of the validity of the WST as a measure of balance performance among older adults. Future studies should focus on expanding from the present by examining the validity of the WST in different populations of older adults, for example, those presenting with limited mobility, or those with conditions/diseases affecting balance. Future studies should also be conducted to develop cut-points/categories for balance level (e.g., "poor", "average", "superior") based on WST scores. This is because the final WST score is expressed as a percentage, with $0 \%$ being the worst balance score and $100 \%$ being the best balance score. The development of such cutpoints/categories could improve the quality of screenings conducted in clinical and community settings and therefore, decision making.

In conclusion, our findings demonstrated a strong inverse association between WST scores and sway area, an important balance metric assessed using a force platform. Although a moderate inverse association was observed between WST scores and sway velocity, such association was not statistically significant. The Wii apparatus is easy to use and cost-effective method that can be used in different settings for assessing balance perfor $\neg$ mance in older adults.

\section{References}

Al-Aama, T. (2011). Falls in the elderly: Spectrum and prevention. In Canadian Family Physician, 57(7),
771-776.

Alexander, B. H., Rivara, F. P., \& Wolf, M. E. (1992). The cost and frequency of hospitalization for fall- 
related injuries in older adults. American Journal of Public Health, 82(7), 1020-1023. https://doi. org/10.2105/AJPH.82.7.1020

Blum, L., \& Korner-Bitensky, N. (2008). Usefulness of the Berg Balance Scale in stroke rehabilitation: A systematic review. Physical Therapy, 88(5),559566. https://doi.org/10.2522/ptj.20070205

Clark, R. A., Bryant, A. L., Pua, Y., McCrory, P., Bennell, K., \& Hunt, M. (2010). Validity and reliability of the Nintendo Wii Balance Board for assessment of standing balance. Gait and Posture, 31(3),307-310. https://doi.org/10.1016/j.gaitpost.2009.11.012

Cohen, J. (2013). Statistical Power Analysis for the Behavioral Sciences (Revised ed.). Academic Press. https://doi.org/10.4324/9780203771587

Jorgensen, M. G., Hansen, N. B., Perez, A. L. R., \& Spaich, E. G. (2014). A standard low-cost worldwide accessible nintendo Wii balance test can differentiate older fallers from non-fallers. In: Jensen W., Andersen O., Akay M. (eds). Replace, Repair, Restore, Relieve - Bridging Clinical and Engineering Solutions in Neurorehabilitation. Biosystems and Biorobotics, 7. Springer, Cham. https://doi. org/10.1007/978-3-319-08072-7_70

Jorgensen, M. G., Laessoe, U., Hendriksen, C., Nielsen, O. B. F., \& Aagaard, P. (2014). Intrarater reproducibility and validity of Nintendo Wii balance testing in community-dwelling older adults. Journal of Aging and Physical Activity, 22(2),269-275. https://doi. org/10.1123/JAPA.2012-0310

La Porta, F., Caselli, S., Susassi, S., Cavallini, P., Tennant, A., \& Franceschini, M. (2012). Is the berg balance scale an internally valid and reliable measure of balance across different etiologies in neurorehabilitation? A revisited rasch analysis study. Archives of Physical Medicine and Rehabilitation, 93(7),12091216. https://doi.org/10.1016/j.apmr.2012.02.020

Leach, J. M., Mancini, M., Peterka, R. J., Hayes, T. L., \& Horak, F. B. (2014). Validating and calibrating the Nintendo Wii balance board to derive reliable center of pressure measures. Sensors, 14(10),1824418267. https://doi.org/10.3390/s141018244

Negus, J. J., Cawthorne, D., Clark, R., Negus, O., Xu, J., March, P. L., \& Parker, D. (2019). Validity and reliability of the Nintendo Wii Fit Stillness score for assessment of standing balance. Asia-Pacific Journal of Sports Medicine, Arthroscopy, Rehabilitation and Technology, 15(2019 January),29-34. https:// doi.org/10.1016/j.asmart.2018.09.001

Park, D. S., \& Lee, G. (2014). Validity and reliability of balance assessment software using the Nintendo Wii balance board: Usability and validation. Journal of NeuroEngineering and Rehabilitation, 11(1),99. https://doi.org/10.1186/1743-0003-11-99

Sterling, D. A., O'Connor, J. A., \& Bonadies, J. (2001). Geriatric falls: Injury severity is high and disproportionate to mechanism. Journal of Trauma - Injury, Infection and Critical Care, 50(1), 116-119. https:// doi.org/10.1097/00005373-200101000-00021

Trombetti, A., Reid, K. F., Hars, M., Herrmann, F. R., Pasha, E., Phillips, E. M., \& Fielding, R. A. (2016). Age-associated declines in muscle mass, strength, power, and physical performance: impact on fear of falling and quality of life. Osteoporosis International, 27(2), 463-471. https://doi.org/10.1007/ s00198-015-3236-5 